\title{
Utilização de oxigenação por membrana extracorpórea (ECMO) no choque cardiogênico refratário: relato de caso e revisão da literatura
}

\author{
Utilization of extracorporeal membrane oxygenation (ECMO) in the cardiogenic refractory \\ shock: case report and literature review
}

Evelyn Sue Nakahira ${ }^{1}$, Filomena Regina Barbosa Gomes Galas ${ }^{2}$

\begin{abstract}
Nakahira ES, Galas FRBG. Utilização de oxigenação por membrana extracorpórea (ECMO) no choque cardiogênico refratário: relato de caso e revisão da literatura / Utilization of extracorporeal membrane oxygenation (ECMO) in the cardiogenic refractory shock: case report and literature review. Rev Med (São Paulo). 2016 out.-dez.;95(4):168-74.
\end{abstract}

RESUMO: Paciente de 29 anos, do sexo masculino, com miocardiopatia dilatada, evoluiu com insuficiência cardíaca refratária ao tratamento clínico. Para manter parâmetros hemodinâmicos foi necessário utilizar balão intra-aórtico e, posteriormente, instalação de oxigenação por membrana extracorpórea (ECMO) como ponte para transplante cardíaco. A ECMO permitiu estabilização, melhora das disfunções orgânicas de tal forma que o paciente chegasse ao transplante em condições hemodinâmicas adequadas. O paciente foi transplantado, evoluiu hemodinamicamente estável no pós-operatório. Duas semanas após ter saído da unidade de terapia intensiva (UTI), recebeu alta.

Descritores: Oxigenação por membrana extracorpórea; Insuficiência cardíaca; Balão intra-aórtico.

\begin{abstract}
A 29 years old male patient, with dilated cardiomyopathy, evolved with refractory heart failure to clinical treatment. To maintain hemodynamic parameters, the use of intra-aortic balloon and there after extracorporeal membrane oxygenation (ECMO) were necessary to bridge the patient to cardiac transplantation. ECMO allowed stabilization, improvement from the organs dysfunctions in a manner that the patient could be submitted to transplantation in adequate hemodynamic conditions. The patient was transplanted, evolved hemodynamically stable in the postoperative. The patient was dischared after two weeks that he had left the intensive care unit.
\end{abstract}

Keywords: Extracorporeal membrane oxygenation; Heart failure; Intra-aortic balloon pumping.

O trabalho é vinculado à Liga de Anestesiologia, Dor e Terapia Intensiva da FMUSP.

1. Faculdade de Medicina da Universidade de São Paulo. E-mail: evelyn.nakahira@fm.usp.br.

2. Instituto do Coração da Faculdade de Medicina da Universidade de São Paulo. E-mail: filomenagalas@hotmail.com.

Endereço para correspondência: Filomena Regina Barbosa Gomes Galas. Av. Dr. Enéas de Carvalho Aguiar, 44 CEP: 05403-000 -

Cerqueira César, São Paulo, Brasil. E-mail: filomenagalas@hotmail.com. 


\section{INTRODUÇÃO}

suporte circulatório mecânico vem sendo cada
vez mais utilizado no manejo da disfunção ventricular de pacientes críticos em combinação com o tratamento convencional da insuficiência cardíaca grave. Estes dispositivos de suporte podem ser classificados de acordo com o tipo (ponte para recuperação; ponte para transplante; ponte para decisão; ponte para terapia de destino); ou de acordo com a duração (de curta duração; os de média; e os de longa duração). A escolha do suporte circulatório depende das condições clínicas e do grau de reversibilidade da disfunção ventricular. Dos dispositivos de curta duração, o balão intra-aórtico tem sido utilizado com frequência em pacientes com baixo débito cardíaco. Em pacientes com baixo débito refratário ao uso do balão, a utilização de oxigenação por membrana extracorpórea (ECMO) é considerada. A ECMO tem sido, nesses casos, utilizada como ponte para recuperação do choque ou como ponte para transplante cardíaco. Apresentamos relato de caso em que o suporte circulatório com ECMO foi importante para a recuperação do paciente.

\section{RELATO DE CASO}

Paciente do sexo masculino, 29 anos, portador de miocardiopatia dilatada, com cansaço e dispneia, apresentava-se em classe funcional I da New York Heart Association (NYHA), em uso de espironolactona; carvedilol; enalapril; digoxina; hidralazina; e furosemida. Após ficar 3 dias sem receber carvedilol e digoxina, apresentou progressão da dispneia seguida de piora da classe funcional para classe funcional IV. Nesta ocasião procurou o pronto socorro, apresentando sintomas de descompensação da insuficiência cardíaca, sendo internado para compensação do quadro clínico. Durante a internação hospitalar evoluiu em choque cardiogênico de difícil controle necessitando doses progressivas de inotrópicos (dobutamina 15,5mcg/kg/min). Apresentou também piora da função renal. No quarto dia de internação evoluiu com hipotensão arterial, náuseas e vômitos seguido de rebaixamento do nível de consciência, cianose central e parada cardio-respiratória (PCR) em ritmo de fibrilação ventricular. Paciente retornou ao ritmo sinusal após 2 ciclos de reanimação cardiopulmonar (RCP), com abertura ocular e movimentação de membros. Foi aumentada a dose de dobutamina, associada epinefrina para melhora do débito cardíaco e controle da hipotensão arterial. Houve piora progressiva da instabilidade hemodinâmica sendo necessário a passagem de balão intra aórtico. Após a passagem do balão intra aórtico houve melhora da pressão arterial e do débito cardíaco. O paciente estabilizou, sendo extubado no dia seguinte, sem sequelas neurológicas. No $11^{\circ}$ dia de internação foi retirado o balão intra-aórtico, seguido da redução gradual de dose de dobutamina. Houve piora progressiva da função renal necessitando aumento da dobutamina para $20 \mathrm{mcg} / \mathrm{kg} / \mathrm{min}$. Apesar dessa medida, o débito cardíaco e a pressão arterial não foram recuperadas. Foi introduzido o balão intra-aórtico, com otimização de fármacos inotrópicos e vasoativos sem necessidade de reintubação. O paciente permaneceu em choque cardiogênico refratrário, sem melhora do débito cardíaco. Devido à manutenção do quadro clínico desfavorável, optou-se por instalação de ECMO veno-arterial periférica como ponte para transplante cardíaco. A ECMO venoarterial foi instalada por punção da veia femoral direita e da artéria femoral esquerda. O procedimento foi realizado sem intercorrências, houve melhora significativa do débito cardíaco o que possibilitou o desmame dos fármacos vassopressores e a diminuição dos inotrópicos. Após estabilização do quadro, paciente foi extubado, permaneceu sem outras disfunções orgânicas, e foi colocado na lista de transplante cardíaco como prioridade. No dia 31/05/2016, o paciente foi submetido a transplante cardíaco ortotópico bicaval. Foi realizada anestesia balanceada, utilizado cetamina, fentanil, pancurônio, midazolan e sevoflurano em doses habituais. Foi utilizado óxido nítrico na retirada da circulação extracorpórea para controle da hipertensão pulmonar. O tempo de tempo de isquemia total foi de 153 minutos. De intercorrências na cirurgia, apresentou dificuldade de sair de circulação extra-corpórea (CEC) por disfunção de enxerto, revertida com ajuste metabólico, volêmico e de inotrópicos/vasopressores. Apresentou fibrilação ventricular na saída da CEC, revertida com cardioversão externa. $\mathrm{O}$ paciente foi extubado e teve o balão intra-aórtico retirado um dia após o transplante cardíaco. No segundo pós-operatório, apresentou aumento da pressão venosa central e disfunção do ventrículo direito, sendo necessário associação de milrinone $0,2 \mathrm{mcg} / \mathrm{kg} /$ min, à dobutamnina $20 \mathrm{mg} / \mathrm{kg} / \mathrm{min}$ e adrenalina $0,2 \mathrm{mcg} /$ kg/min para otimização dos parâmetros hemodinâmicos e redução de pressão venosa central. No $13^{\circ}$ dia após o transplante, foram retirados os fármacos vasoativos. No $15^{\circ}$ pós-operatório recebeu alta da unidade de terapia intensiva (UTI). Paciente apresentou boa evolução e recebeu alta da enfermaria duas semanas após a alta da UTI. O paciente encontrava-se estável hemodinamicamente e recebeu alta hospitalar (Figura 1). 

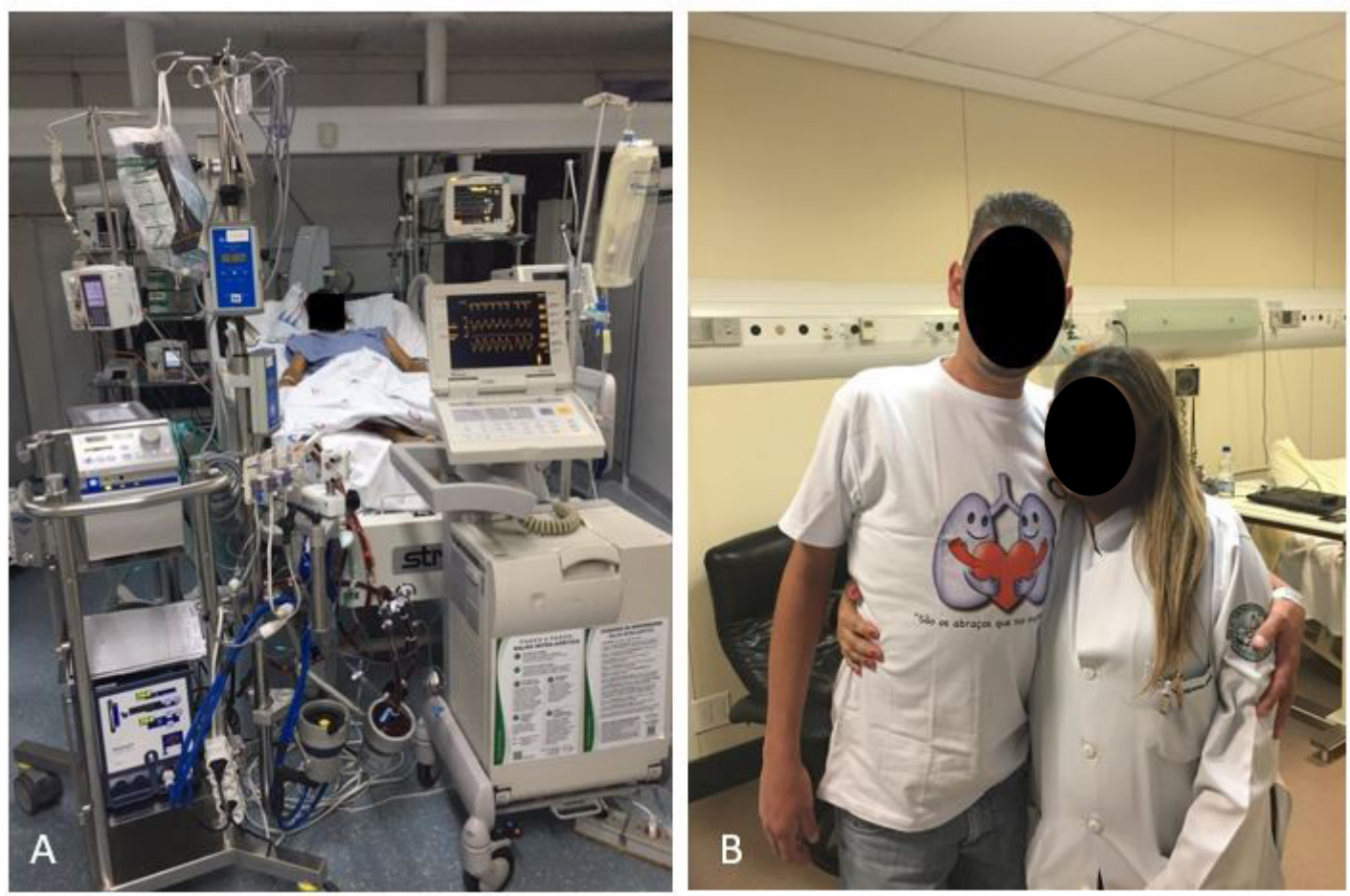

Figura 1. A. Paciente do caso apresentado em ECMO com as canulações visíveis na fotografia. B. Paciente no dia da alta (Fotografias cedidas pela Dra. Filomena Regina Barbosa Gomes Galas)

\section{DISCUSSÃO}

\section{Utilização da assistência ventricular no choque cardiogênico refratário}

O choque cardiogênico refratário pode ser definido como disfunção de órgão atribuível a débito cardíaco reduzido e insuficiente, apesar da administração de altas doses de fármacos inotrópicas e vasopressoras. São causas de choque cardiogênico: infarto do miocárdio, miocardite fulminante, exacerbação de insuficiência cardíaca crônica, arritmias intratáveis, insuficiência cardíaca pós cardiomiotomia, intoxicação por drogas, entre outras $^{1}$. Apesar da alta mortalidade associada ao choque cardiogênico refratário, houve mudança do prognóstico desses pacientes com a introdução de dispositivos de assistência ventricular. Nos pacientes pediátricos, estima-se que 1 a 5\% necessitarão de algum tipo de suporte circulatório mecânico antes ou depois da cirurgia cardíaca. O desenvolvimento de dispositivos mais seguros e mais simples vem aumentando a indicação de suporte circulatório, melhorando os resultados clínicos, além de gerar uma maior necessidade de capacitação profissional para sua utilização e manejo. Esses números podem ser mais expressivos nos serviços com programa de transplante bem desenvolvido.

A assistência circulatória possui como objetivos: prevenção ou interrupção do declínio progressivo da função ventricular; promoção da recuperação ventricular; possibilitar o tratamento; prevenção e minimização da disfunção orgânica; e redução de mortes. Assim, a assistência circulatória pode ser vista como ponte para recuperação e/ou candidatura e/ou transplante e/ou terapia de destino. Com o desenvolvimento de dispositivos de maior segurança e simplicidade, ocorre aumento dos casos em que a assistência circulatória é indicada, com melhores resultados sendo obtidos.

\section{Dispositivos de curta duração}

O suporte circulatório temporário se refere a um grupo de dispositivos geralmente utilizados durante menos de 30 dias para compensar falência do mecanismo de bomba do coração. O primeiro dispositivo utilizado com essa finalidade foi o balão intra-aórtico. São indicações do suporte circulatório temporário: ponte para recuperação; ponte para decisão (prognóstico incerto) ${ }^{2}$; ponte para dispositivos de longa permanência; ponte para transplante cardíaco $^{2,3}$; terapia de destino ${ }^{4}$.

A escolha do dispositivo circulatório temporário é importante para limitar iatrogenias agudamente, mas também interfere nas possibilidades de terapias se não ocorrer recuperação cardíaca ${ }^{3}$. 


\section{Balão intra-aórtico}

É o tipo de suporte circulatório mais utilizado. É implantado percutaneamente através da veia femoral (acesso mais usual). Consiste em um balão preenchido por hélio que insufla na luz da aorta descendente durante a diástole, produzindo um aumento na pressão diastólica na raiz da aorta e permite com que a aumente a perfusão das coronárias. Na sístole, o balão se esvazia, cria efeito de vácuo e permite redução da pós-carga, reduzindo o consumo de oxigênio pelo miocárdio. É o único dos suportes circulatórios temporários que melhora a perfusão coronariana. No entanto, não gera aumentos significativos na perfusão tecidual periférica ou no débito cardíaco ${ }^{2}$.

\section{Impella}

Impella consiste em uma hélice posicionada na saída do ventrículo esquerdo para a aorta ascendente e que utiliza o princípio de Arquimedes. A rotação de uma hélice inserida em um tubo oco bombeia sangue do ventrículo esquerdo para a aorta, sendo esse fluxo de sangue adicional contínuo. É posicionado por via percutânea (acesso pela artéria femoral). Esse dispositivo melhora a perfusão tecidual, a pressão arterial, reduz a pressão de capilar pulmonar, reduz o volume sistólico e reduz a carga do ventrículo esquerdo, reduzindo o consumo de oxigênio do miocárdio. Há grande aumento no débito cardíaco ofertado, que pode chegar a 3,3L/min nos modelos de implante percutâneo ${ }^{2}$.

\section{TandemHeart}

Consiste em uma bomba extracorpórea que pode substituir tanto o coração direito, quanto o esquerdo ou ambos. Para assistência esquerda, uma cânula posicionada na veia femoral vai até o átrio esquerdo (perfurando o septo atrial), onde aspira sangue e o devolve para a artéria femoral. Consiste em uma bomba que trabalha em paralelo ao coração, diferentemente do Impella, que é em série ao coração. Para assistência direita, aspira do átrio direito, e devolve o sangue na artéria pulmonar. O quanto de fluxo que consegue dar é dependente do tamanho da cânula. Cânulas de $15 \mathrm{Fr}$ dão $3,51 / \mathrm{min}$ de fluxo adicional, cânulas de $19 \mathrm{Fr}$ dão mais que $51 / \mathrm{min}$ de fluxo adicional. É capaz de aumentar a perfusão tecidual e reduzir o consumo de oxigênio do miocárdio ao reduzir a demanda ventricular ${ }^{2}$.

\section{CentriMag ${ }^{\circledR}$}

Consiste em um dispositivo que oferece fluxo contínuo através de uma bomba extracorpórea, e é um dos mais comuns dispositivos cirurgicamente implantados para suporte circulatório temporário. A implementação é através de esternotomia mediana. A bomba contém um Impella, com levitação magnética, com o objetivo de minimizar hemólise. Pode ser utilizado para suporte ventricular direito, esquerdo e biventricular. Para suporte do coração esquerdo, uma cânula é implanta no ventrículo esquerdo e o sangue é devolvido em cânula implanta na aorta com fluxo de 5 a $7 \mathrm{~L} / \mathrm{min}^{2}$.

\section{Abiomed BVS 5000}

Bomba cirurgicamente implantada que dá suporte ao ventrículo direito ou ao ventrículo esquerdo ou aos dois. O fluxo é de forma pulsátil e promovido por mecanismo pneumático. A bomba é composta por duas câmaras, uma atrial de preenchimento gravitacional, que é conectada a uma ventricular, que dá fluxo adicional de $6 \mathrm{~L} / \mathrm{min}$ de sangue. Para suporte esquerdo, uma cânula é implantada no átrio esquerdo, com devolução do sangue em cânula na aorta torácica. Para suporte direito, uma cânula é implantada no átrio direito, como cânula para devolução de sangue implantada na artéria pulmonar. Ainda é de uso limitado $^{2}$.

\section{Rotaflow $^{\circledR}$}

Dispositivo semelhante ao CentriMag e ao Impella, funciona propulsionando continuadamente o sangue. Implantado através de esternotomia mediana ${ }^{5}$.

\section{Dispositivos de longa duração}

Em casos em que não há recuperação cardíaca após súbita falência cardíaca ou de insuficiência cardíaca crônica, podem ser utilizados dispositivos de assistência ventricular implantáveis e de longa permanência. Os corações totalmente artificiais ainda são utilizados raramente, mas é esperado que a sua aplicação aumente com os anos ${ }^{3}$.

Os dispositivos percutâneos (PVADs) permitem o alívio efetivo da sobrecarga ventricular na emergência, enquanto resultam em pressão de perfusão sistêmica capaz de preservar as funções orgânicas. Os dispositivos implantáveis paracorpóreos ou definitivos cirurgicamente implantáveis (LVADs, RVADs, BiVADs) resultam em aumento da sobrevida dos pacientes com choque cardiogênico. Estes dispositivos têm sido utilizados com maior frequência nestes pacientes, e estão relacionados à diminuição da mortalidade.

\section{Berlin Heart EXCOR ${ }^{\circledR}$}

É dispositivo de assistência circulatória paracorpórea pulsátil pneumático com cânulas que conectam as bombas a câmaras cardíacas e grandes vasos. Com mais de 2371 implantes no mundo, é de fácil visualização o desempenho da bomba. Há tamanhos especiais para crianças. Pode ser de suporte uni ou biventricular. Para suporte à esquerda, uma cânula implantada cirurgicamente no ápice do ventrículo esquerdo retira sangue para a bomba e, o sangue é devolvido em cânula implantada na aorta. Para suporte circulatório à direita, uma cânula implantada no átrio direito leva 0 sangue para a bomba e uma cânula devolve o sangue para a artéria pulmonar. No caso do Berlin Heart EXCOR, as cânulas são cirurgicamente inseridas e tuneilizadas até a pele já que a bomba se encontra localizada fora do corpo do paciente ${ }^{6}$ (Figura 2). 


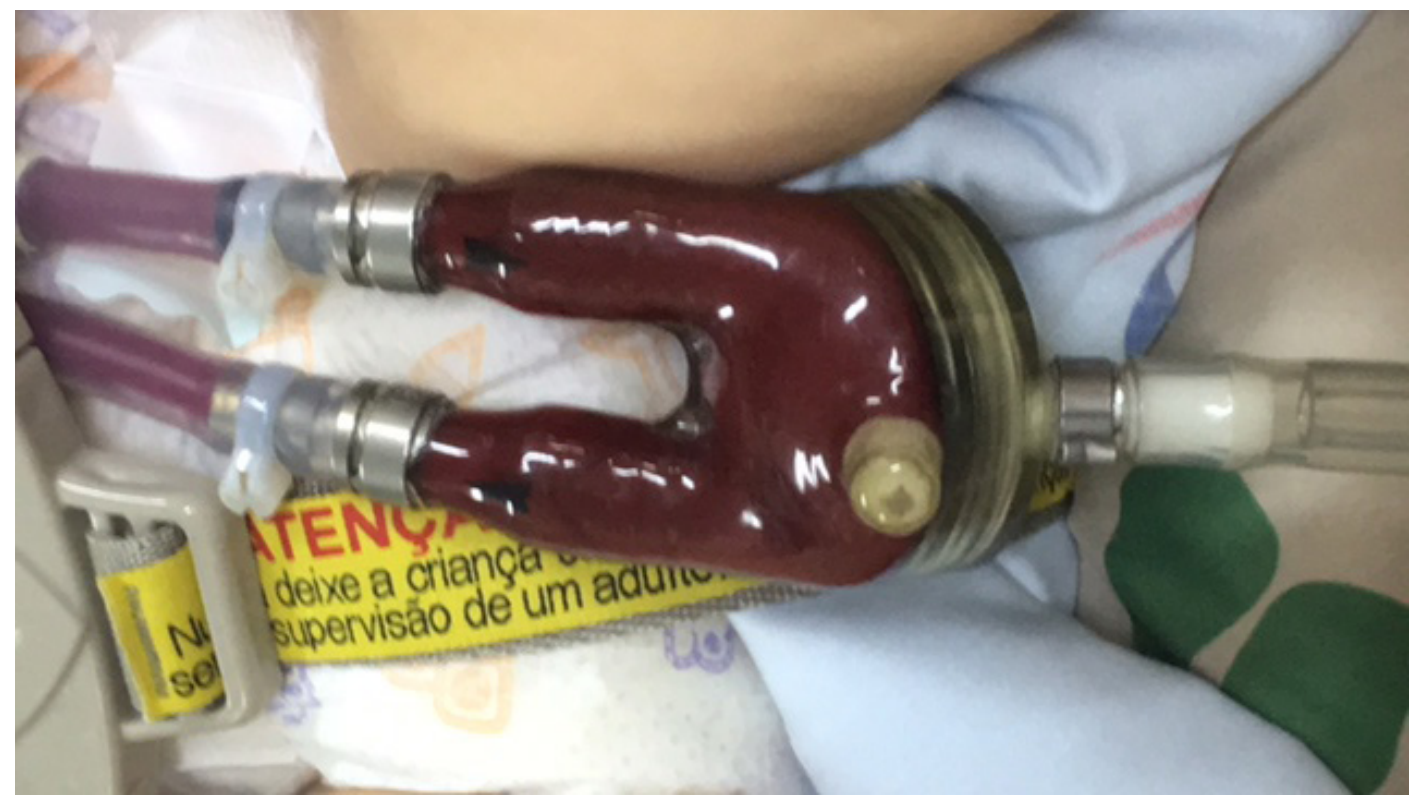

Figura 2. Fotografia do dispositivo Berlin Heart EXCOR (Fotografia cedida pela Dra. Filomena Regina Barbosa Gomes Galas)

\section{Thoratec pVAD ${ }^{\circledR}$}

Bomba cirurgicamente implantada que dá suporte ao ventrículo direito ou ao ventrículo esquerdo ou aos dois. A bomba é de mecanismo pneumático e pulsátil. O ritmo da bomba é determinado pelo eletrocardiograma, ou de forma fixa, ou de acordo com o enchimento do dispositivo de assistência ventricular. A bomba é posicionada fora do corpo, na parede abdominal anterior, disso pode ser utilizada em pacientes de quase todos os tamanhos. Para suporte ventricular esquerdo, a cânula de influxo é implantada no ápice do ventrículo esquerdo e, a cânula que devolve o sangue, na aorta descendente. Para suporte ventricular direito, a cânula de influxo é implantada no átrio direito e, a cânula que devolve o sangue, na artéria pulmonar. Pode ofertar fluxo de 1,3 a 7,2 L/min ${ }^{2,4}$.

\section{Berlin Heart INCOR ${ }^{\circledR}$}

É uma bomba que se caracteriza por possuir um propulsor de mecanismo magnético. A sua aplicação é para suporte ventricular esquerdo, com cânula implantada no ápice do ventrículo esquerdo que retira destina o sangue para a bomba e cânula de inserção na aorta ascendente que devolve o sangue. A bomba é totalmente inserida cirurgicamente no paciente, apenas com exteriorização de cabos que ofertam energia para a bomba. Oferece fluxo de sangue de até 10 litros por minuto ${ }^{7}$. O Sistema INCOR $^{\circledR}$ foi projetado com o objetivo de função de longo prazo, conforme necessário para uso como terapia permanente, na medida em que pode assumir o trabalho do ventrículo esquerdo sem desgaste. Berlin Heart INCOR $^{\circledR}$ também pode ser implantado como ponte para o transplante e ponte para a recuperação.

\section{Heart Mate (II e III) ${ }^{\circledR}$}

HeartMate II consiste em dispositivo de assistência ventricular implantável, tem fluxo contínuo de até $10 \mathrm{~L} / \mathrm{min}$ em pressão média de $100 \mathrm{mmHg}$. Consiste em uma cânula implantada no ápice do ventrículo esquerdo que direciona o sangue para a bomba implantada pré-peritonealmente e, dessa, o sangue é devolvido para a circulação através de uma cânula implantada na aorta. Esse sistema de suporte circulatório é conectado percutaneamente a monitor e fonte de energia, localizados externamente ao paciente ${ }^{8}$.

HeartMate III é um dispositivo de assistência ventricular totalmente implantável. Tem mecanismo de propulsão por levitação magnética, com cânula inserida no ápice do ventrículo esquerdo que leva o sangue para o dispositivo e cânula inserida na aorta que devolve o sangue para a circulação sistêmica. A bomba é inserida intrapericardialmente. Externamente, o paciente permanece com as baterias do dispositivo, que são conectadas à bomba por fios.

\section{SynCardia $^{\circledR}$}

Consiste em coração artificial total, uma modalidade de assistência circulatória ainda pouco utilizada clinicamente, o Syncardia é o único modelo com experiência clínica em uso. Consiste em duas câmaras de propulsão pneumática, com fluxo pulsátil e que oferece fluxo sistêmico e pulmonar. Oferece fluxo de até 9,5 L/ min. O dispositivo implantado pesa $160 \mathrm{~g}$, o driver com baterias extracorpóreas pesa, na sua versão portátil, $6,1 \mathrm{~kg}^{3}$. 


\section{ECMO}

\section{Considerações Gerais}

Em 1812, surgiu o primeiro modelo de circulação extra-corpórea, proposta por Le Gallois. Em 1955 foi desenvolvida a primeira membrana oxigenadora por Kolff e Balzer ${ }^{10}$. Esse desenvolvimento tecnológico permitiu que, em 1972, ocorresse a primeira aplicação de ECMO no atendimento de um paciente pós-trauma. Desde então, o número de pacientes em ECMO é crescente.

São componentes essenciais da ECMO: acesso vascular, cânulas, bomba e membrana oxigenadora.

O sucesso da ECMO requer uma equipe multidisciplinar, treinada, com experiência nessa tecnologia. A equipe é composta geralmente por cirurgiões cardiotorácicos, perfusionistas, intensivistas, anestesiologistas e profissionais de cuidados respiratórios ${ }^{1}$.

\section{Indicações de ECMO}

São situações que a ECMO pode ser utilizada para melhora da condição clínica do paciente: parada cardiorrespiratória não responsiva a outras manobras de ressuscitação, falência circulatória primária, falência respiratória com grave disfunção cardíaca.

No caso de insuficiência cardíaca refratária deve se considerar ECMO quando o paciente apresenta miocardite associada a choque cardiogênico, miocardite fulminante, depressão miocárdica associada à sepse, em casos em que o paciente evolui para ressuscitação corpórea cardiopulmonar extracorpórea; falência de enxerto após transplante cardíaco ${ }^{11}$. ECMO é tratamento de ponte para implantação de outros dispositivos de assistência circulatória ou transplante cardíaco ${ }^{11}$. Assim, pacientes com função cardíaca não recuperável, que não são candidatos para dispositivo de assistência ventricular ou transplante cardíaco, não devem ser selecionados para $\mathrm{ECMO}^{1}$.

É necessário considerar que a ECMO, por devolver sangue em território arterial, aumenta o esforço que o ventrículo esquerdo precisa fazer para bombear sangue, com aumento do consumo de oxigênio. Isso pode ser minimizado pela implementação de BIAO ou Impella² .

\section{REFERÊNCIAS}

1. Lawler PR, Silver DA, Scirica BM, Couper GS, Weinhouse GL, Camp PC Jr. Extracorporeal membrane oxygenation in adults with cardiogenic shock. Circulation. 2015;131(7):676-80. doi: http://dx.doi.org/10.1161/ CIRCULATIONAHA.114.006647.

2. Saffarzadeh A, Bonde P. Options for temporary mechanical circulatory support. J Thorac Dis. 2015;7(12):2102-11. doi: http://dx.doi.org/10.3978/j.issn.2072-1439.2015.09.14.

\section{Choque cardiogênico e ECMO}

No choque cardiogênico refratário a outros tratamentos, a ECMO arterial-venosa é atualmente a escolha para assistência circulatória já que não depende do débito cardíaco do paciente ${ }^{1}$. Entre os pontos considerados estão: canulação fácil e rápida; não necessita de esternotomia ou cardiotomia; anestesia local para sua implementação; pode ser utilizado em situações de emergência; provê fluxo alto e débito cardíaco estável; permite suporte cardiopulmonar através da bomba centrífuga e do oxigenador.

\section{Ponte para transplante}

Dispositivos de assistência circulatória temporária podem ser utilizados como ponte para decisão de terapia definitiva em insuficiência cardíaca aguda rapidamente progressiva e em que a morte ocorrerá sem o uso de dispositivos de assistência circulatória. Disso, reduz a disfunção de órgãos e pode permitir com que pacientes inelegíveis ao transplante cardíaco se tornem possíveis receptores. Pacientes com alto risco de morte também podem ser submetidos à terapia de assistência circulatória temporária até que um órgão compatível esteja disponível ${ }^{12}$.

\section{Complicações da ECMO}

Alguns pacientes em ECMO podem apresentar complicações, como sangramento, isquemia de membros inferiores (se cânulas em femorais), trombose, embolia sistêmica, coagulação intravascular disseminada (CIVD); hemólise; trombocitopenia; acidente vascular cerebral ${ }^{1,13}$.

\section{CONCLUSÕES}

Neste caso apresentado, a utilização da ECMO e o cuidado sistematizado foram fundamentais para que o transplante cardíaco fosse realizado com sucesso e o paciente tivesse alta hospitalar bem sem outras comorbidades. A ECMO e outras formas de assistência circulatórias devem ser consideradas em pacientes graves para ponte para recuperação, ou decisão, ou terapia de destino, ou transplante.
3. Shekar K, Gregory SD, Fraser JF. Mechanical circulatory support in the new era: an overview. Crit Care. 2016;20:66. doi: http://dx.doi.org/10.1186/s13054-016-1235-3

4. Peura JL, Colvin-Adams M, Francis GS, Grady KL, Hoffman TM, Jessup M, et al. Recommendations for the use of mechanical circulatory support : device strategies and patient selection a scientific statement from the American Heart Association. Circulation. 2012;126:2648-67. doi: http://dx.doi.org/10.1161/CIR.0b013e3182769a54. 
5. Khani-Hanjani A, Loor G, Chamogeorgakis T, Shafii A, Mountis M, Hanna M, et al. Case series using the ROTAFLOW system as a temporary right ventricular assist device after HeartMate II implantation. ASAIO J. 2013;59(4):456-60. doi: http://dx.doi.org/10.1097/ MAT.0b013e318291d133

6. Hetzer R, Alexi-Meskishvili V, Weng Y, Hübler M, Potapov E, Drews T, et al. Mechanical cardiac support in the young with the Berlin Heart EXCOR pulsatile ventricular assist device : 15 years experience. Semin Thorac Cardiovasc Surg Pediatr Card Surg Annu. 2006;99-108. doi: http://dx.doi. org/10.1053/j.pcsu.2006.02.012.

7. Schmid C, Tjan TDT, Etz C, Schmidt C, Wenzelburger F, Wilhelm M, et al. First clinical experience with the Incor left ventricular assist device. J Heart Lung Transplant. 2005;24(9):1188-94. doi: http://dx.doi.org/10.1016/j. healun.2004.08.024.

8. John R, Kamdar F, Liao K, Colvin-Adams M, Boyle A, Joyce L. Improved survival and decreasing incidence of adverse events with the heartmate ii left ventricular assist device as bridge-to-transplant. Ann Thorac Surg. 2008;86(4):122735. doi: http://dx.doi.org/10.1016/j.athoracsur.2008.06.030.
9. Schmitto JD, Hanke JS, Rojas SV, Avsar M, Haverich A. First implantation in man of a new magnetically levitated left ventricular assist device (HeartMate III). J Hear Lung Transplant. 2015;34(6):858-60. doi: http://dx.doi. org/10.1016/j.healun.2015.03.001.

10. Kolff WJ, Balzer R. The artificial coil lung. Trans Am Soc Artif Int Organs. 1955;1:39-42.

11. Kulkarni T, Sharma NS, Diaz-Guzman E. Extracorporeal membrane oxygenation in adults. Clev Clin J Med. 2016;83(5):373-84. doi: http://dx.doi.org/10.3949/ ccjm.83a.15021

12. Jasseron $C$, Lebreton $G$, Cantrelle $C$, Legeai C, Leprince $P$, Flecher $\mathrm{E}$, et al. Impact of heart transplantation on survival in patients on venoarterial extracorporeal membrane oxygenation at listing in France. Transplantaion. 100(9):197987. doi: http://dx.doi.org/10.1097/TP.0000000000001265.

13. Stallion A, Cofer BR, Rafferty JA, Ziegler MM, Ryckman FC. The significant relationship between platelet count and haemorrhagic complications on ECMO. Perfusion. 1994;9(4):265-9. doi: 10.1177/026765919400900404. 